Pacific Journal of Mathematics

ON ASYMPTOTIC PRIME DIVISORS 


\title{
ON ASYMPTOTIC PRIME DIVISORS
}

\author{
L. J. RATLIFF, JR.
}

Several results are proved concerning the set $\hat{A}^{*}(I)=\{P \in \operatorname{Spec} R$; $P$ is a prime divisor of the integral closure $\left(I^{t}\right)_{a}$ of $I^{l}$ for all large $\left.i\right\}$, where $I$ is an ideal in a Noetherian ring $R$. Among these are: if $P$ is a prime divisor of $\left(I^{l}\right)_{a}$ for some $i \geq 1$, then $P$ is a prime divisor of $\left(I^{n}\right)_{a}$ for all $n \geq i$; a characterization of Cohen-Macaulay rings and of altitude two local UFDs in terms of $\hat{A}^{*}(I)$; and, some results on the relationship of $\hat{A}^{*}(I)$ to $\hat{A}^{*}(I S)$ with $S$ a flat $R$-algebra and to $\hat{A}^{*}((I+z) / z)$ with $z$ a minimal prime ideal in $R$.

1. Introduction. The sets $\operatorname{Ass}\left(R / I^{i}\right)$ and $\operatorname{Ass}\left(R /\left(I^{i}\right)_{a}\right)$ have been studied in several recent papers, where $I$ is an ideal in a Noetherian ring $R$. For example, in [14, (2.5)] and [5, Prop. 7] it was shown that if height $I \geq 1$, then the sets $\operatorname{Ass}\left(R /\left(I^{i}\right)_{a}\right)$ are equal for all large $i$. Calling this set $\hat{A}^{*}(I)$, it was shown in $[\mathbf{1 4},(2.6 .1)]$ that $\hat{A}^{*}(I)$ is contained in the sets $\operatorname{Ass}\left(R / I^{i}\right)$ for all large $i$. Also, in [1] and in [5, Corol. 5] it was shown that these latter sets are also equal for all large $i$, even without the assumption that height $I \geq 1$. Further, in [15, Thm. 1] it was shown that there exists a positive integer $i$ such that the maximal ideal $M$ in a local domain $R$ is in $\operatorname{Ass}\left(R /\left(I_{a}\right)\right.$ ) for all nonzero ideals $I \subseteq M^{i}$ (and hence $M \in \hat{A}^{*}(I)$ for all nonzero ideals $\left.I \subseteq M\right)$ if and only if there exists a height one maximal ideal in the integral closure of $R$, and in [22, Corol. 2.8] it was shown that if $M \in \hat{A}^{*}(I)$ for a given ideal $I$ in $R$, then $M \in \hat{A}^{*}(I J)$ for all nonzero ideals $J$ in $R$. Numerous other such results could be mentioned here, but instead we refer the reader to [7] and to [18] where most of the known results in this area are summarized.

The purpose of this paper is to add several new results to this area and also to strengthen several of the known results. In $\$ 2$ we remove the restriction on height $I \geq 1$ in the above mentioned results, and also show that the sets $\operatorname{Ass}\left(R /\left(I^{l}\right)_{a}\right)$ are monotonically increasing (and eventually constant). In $\S 3$ the monotonicity of the sets $\operatorname{Ass}\left(R / I^{i}\right)$ is considered, and $\S \S 4$ and 5 contain characterizations in terms of $\hat{A}^{*}(I)$ of Cohen-Macaulay rings and of altitude two local UFDs, respectively. Some results showing the relation between the sets $\hat{A}^{*}(I)$ and the analogous sets in $S$ and in $R / z$ are given in $\S 6$, where $S$ is a flat $R$-algebra and $z$ is a minimal prime ideal in $R$, and the final section, $\S 7$, contains some results on these sets and the analytic spread of the ideal $I$. 
I think results on asymptotic prime divisors are quite interesting in their own right, and it turns out that they are also closely connected to some other areas of current research interest, such as: going-down between prime ideals; asymptotic sequences; and the catenary chain conjectures. (See [7] and [18].) Thus knowledge about such prime divisors is of some interest and importance.

2. Basic properties of $\hat{A}^{*}(I)$. In this section it is shown that the basic results concerning $\hat{A}^{*}(I)$ which are known to hold for ideals $I$ such that height $I \geq 1$ actually hold for all ideals $I$ in all Noetherian rings. Then it is shown that the sets $\operatorname{Ass}\left(R /\left(I^{i}\right)_{a}\right)$ are monotonically increasing (and eventually constant).

We begin by listing a few notational conventions and definitions.

(2.1) Definitions. Throughout $R$ is a Noetherian ring (commutative with identity) and $I$ is an ideal in $R$.

(2.1.1) The integral closure of $R$ in its total quotient ring will be denoted $R^{\prime}$.

(2.1.2) If $R$ is local with maximal ideal $M$, then the $M$-adic completion of $R$ will be denoted $R^{*}$.

(2.1.3) If $B$ is a finite $R$-module, then $\operatorname{Ass}(B)$ denotes the set of associated primes of $B$. In particular, $\operatorname{Ass}(R)$ is the set of prime divisors of zero in $R$, and mAss $R$ denotes the subset of minimal prime ideals in $R$.

(2.1.4) The integral closure of $I$ in $R, I_{a}$, is the set of all $x \in R$ satisfying an equation of the form $X^{n}+b_{1} X^{n-1}+\cdots+a_{n}=0$ where $a_{i} \in I^{i}$. Therefore $I_{a}$ is an ideal in $R$ and $I \subseteq I_{a} \subseteq \operatorname{Rad} I$.

(2.1.5) $\hat{A}^{*}(I)=\operatorname{Ass}\left(R /\left(I^{i}\right)_{a}\right)$ for all large $i$. (It is shown in (2.7) that this set is well defined for all ideals $I$.)

(2.1.6) $A^{*}(I)=\operatorname{Ass}\left(R / I^{i}\right)$ for all large $i$. This set is well defined for all ideals $I$ by [1] and [5, Corol. 5].

(2.1.7) $\Re=\Re(R, I)$ denotes the Rees ring of $R$ with respect to $I$. Therefore $\Re$ is the graded subring $R[u, t I]$ of $R[u, t]$, where $t$ is an indeterminate and $u=1 / t$.

Concerning (2.1.7), note that $\Re$ is a Noetherian ring, $u$ is a regular element in $\Re$, and $u^{i} \Re \cap R=I^{i}$ for all $i \geq 1$.

The following lemma and its corollary will be quite useful in this section. Concerning (2.2), it is shown in [12, Corol. 2.12(1)] that if $b$ is a regular nonunit in a Noetherian ring $R$, then $b R^{\prime}$ is a finite intersection of height one primary ideals. 
(2.2) LEMMA. Let I be an ideal in a Noetherian ring $R$, let $\Re=\Re(R, I)$, let $p^{\prime}$ be a (height one) prime divisor of $u \Re^{\prime}$, and let $Q=p^{\prime} \cap R$. Then $t I \subseteq p^{\prime}$ if and only if height $Q=0$.

Proof. Let $z^{\prime}$ be a minimal prime ideal in $\Re^{\prime}$ that is contained in $p^{\prime}$. Then $z=z^{\prime} \cap R$ is a minimal prime ideal in $R$ that is contained in $Q$ and $z^{\prime}=z T \cap \Re^{\prime}$, where $T$ is the total quotient ring of $\Re^{\prime}$ (and of $R[t]$ ). Now $\Re(R / z,(I+z) / z) \cong \Re /\left(z^{\prime} \cap \Re\right)$, by [20, Lemma 1.1], and $\mathcal{R}^{\prime} / z^{\prime}$ is an integrally dependent overring of $R /\left(z^{\prime} \cap \Re\right)$. We consider two cases: (a) height $Q=0$; and (b) height $Q \geq 1$.

If (a) holds then $Q=z$ and $I \subseteq u \Re^{\prime} \cap R \subseteq Q=z$, so $t I \subseteq z T \cap \Re^{\prime}$ $=z^{\prime} \subset p^{\prime}$. Therefore $\Re /\left(z^{\prime} \cap \Re\right)=(R / z)[u]$, so it readily follows that $p^{\prime}=\left(z^{\prime}, u\right) \Re^{\prime}$.

If (b) holds let $\delta=\Re(R / z,(I+z) / z) \cong \Re /\left(z^{\prime} \cap \Re\right)$, and to simplify notation identify these rings. Then $\delta \subseteq \Re^{\prime} / z^{\prime} \subseteq \mathcal{S}^{\prime}$, so there exists a height one prime divisor $P^{\prime}$ of $u \delta^{\prime}$ that lies over $p^{\prime} / z^{\prime}$. Since $Q / z=$ $\left(p^{\prime} / z^{\prime}\right) \cap(R / z) \neq(0)$, it follows from what was shown in the preceding paragraph that $I \not z z$. Therefore height $(I+z) / z \geq 1$, so, by the proof of [10, Lemma 3.2(1)] (from the third sentence on), $t^{*}=\operatorname{trd}\left(\delta^{\prime} / P^{\prime}\right) /(R / Q)$ $\geq 1$, hence $t((I+z) / z) \not P^{\prime}$, since $u \in P^{\prime}$. Therefore it follows that $t I \nsubseteq p^{\prime}$.

(2.3) Corollary. With $R, I$, and $\Re$ as in (2.2), if $P$ is a prime divisor of $\left(u^{i} \Re\right)_{a}$ for some $i \geq 1$, then either height $P \cap R=0$ and $P=$ $((P \cap R) R[t, u] \cap \Re, u) \Re=(u, P \cap R, t I) \Re$ or height $P \cap R \geq 1$ and $t I \not \subset$.

Proof. If $P$ is a prime divisor of $\left(u^{i} \Re\right)_{a}$ for some $i \geq 1$, then there exists a prime divisor $p^{\prime}$ of $u^{i} \Re^{\prime}$ (and of $u \Re^{\prime}$ ) such that $p^{\prime} \cap \Re=P$, since $\left(u^{i} \Re\right)_{a}=u^{i} \Re{ }^{\prime} \cap R$, so the conclusion readily follows from (2.2) and the second paragraph of its proof.

It was shown in $[14,(2.5)]$ that if $I$ is an ideal in a Noetherian ring $R$ such that height $I \geq 1$ and if $P$ is a prime divisor of $\left(I^{i}\right)_{a}$ for some $i \geq 1$, then $P$ is a prime divisor of $\left(I^{n}\right)_{a}$ for all large $n$. (2.4) is a considerable extension of this result. In the proof of (2.4), the following readily shown fact is used, where $R$ is the Rees ring of $R$ with respect to $I:$ if $b t^{k} \in R$, then $\left(u^{i} \Re\right)_{a}: b t^{k} \Re=\left(u^{i+k} \Re\right)_{a}: b \Re$. 
(2.4) TheOREM. (Cf. [14, (2.5)].) Let I be an ideal in a Noetherian ring $R$ and let $Q$ be a prime divisor of $\left(I^{i}\right)_{a}$ for some $i \geq 1$. Then $Q$ is a prime divisor of $\left(I^{n}\right)_{a}$ for all $n \geq i$.

Proof. By hypothesis there exists $b \in R$ such that $\left(I^{i}\right)_{a}: b R=Q$. Let $\Re=\Re(R, I)$. Then $Q \Re=\left(\left(I^{i}\right)_{a}: b R\right) \Re \subseteq\left(I^{i}\right)_{a} \Re: b \mathcal{R} \subseteq\left(u^{i} \Re\right)_{a}: b \Re$ and $\left(\left(u^{i} \mathcal{R}\right)_{a}: b \mathcal{R}\right) \cap R=\left(I^{i}\right)_{a}: b R=Q$, by [23, p. 220], since $\left(u^{i} \Re\right)_{a} \cap$ $R=\left(I^{i}\right)_{a}$. Therefore there exists a prime divisor $P$ of $\left(u^{i} \mathcal{R}\right)_{a}$ such that $\left(u^{i} \Re\right)_{a}: b \Re \subseteq P$ and $P \cap R=Q$. By (2.3), either height $Q=0$ or height $Q \geq 1$ and $t I \nsubseteq P$. If height $Q=0$, the conclusion is clear, so it may be assumed height $Q \geq 1$, so there exists $c \in I$ such that $c t \notin P$. Therefore for all $j \geq 0$ it holds that $\left(u^{i+j} \mathcal{R}\right)_{a}: b c^{j} \mathcal{R}=\left(u^{i} \Re\right)_{a}: b c^{j} t^{j} \mathcal{R} \subseteq$ $P$, and contracting to $R$ it follows that $\left(I^{i+j}\right)_{a}: b c^{j} R=Q$, so $Q$ is a prime divisor of $\left(I^{n}\right)_{a}$ for all $n \geq i$.

(2.5) was proved in [11, Prop. 3.5] for local domains. It is important to extend this result to the local ring case, because its corollary, (2.6), plays an important role in the deeper results in $\$ 6$.

(2.5) Proposition. Let $R$ be a local ring. If there exists a height one maximal ideal in $R^{\prime}$, then there exists a depth one minimal prime ideal in the completion $R^{*}$ of $R$.

Proof. If there exists a height one maximal ideal in $R^{\prime}$, then there exists an element $x$ in $R^{\prime}$ such that $R[x]$ has a height one maximal ideal, so $R^{*}[x]=(R[x])^{*}$ has a height one maximal ideal, say $N^{*}$. Then if $z$ is a minimal prime ideal in $R[x]^{*}$ that is contained in $N^{*}$, then depth $z=$ altitude $R[x]^{*} / z=$ height $N^{*} / z=1$, since $R[x]^{*} / z$ is a complete local domain. Finally, $z \cap R^{*}$ is minimal and depth $z=\operatorname{depth} z \cap R^{*}$ by integral dependence.

It is shown in [15, Thm. 1] that if $R$ is a local ring and there exists a depth one $z \in \mathrm{mAss} R^{*}$, then there exists a positive integer $n$ such that $M$ is a prime divisor of $I_{a}$ for all ideals $I \subseteq M^{n}$ such that height $I \geq 1$, and conversely. This, together with (2.5), is used in the next two results to extend known results to more general cases.

(2.6) Corollary. (Cf. [15, Corol. 2].) Let $b$ be a regular element in a Noetherian ring $R$. Then the following statements hold:

(2.6.1) If $Q^{\prime}$ is a prime divisor of $b R^{\prime}$, then $Q^{\prime} \cap R$ is a prime divisor of $\left(b^{i} R\right)_{a}$ for all large $i$. 
(2.6.2) If $Q$ is a prime divisor of $\left(b^{i} R\right)_{a}$ for some $i \geq 1$, then there exists an integerr $n$ such that $Q$ is a prime divisor of $I_{a}$ for all ideals $I \subseteq Q^{(n)}=$ $Q^{n} R_{Q} \cap R$ such that height $I_{Q} \geq 1$, hence $Q \in \hat{A}^{*}(I)$ for all ideals $I \subseteq Q$ such that height $I_{Q} \geq 1$.

Proof. The proof is essentially the same as the proof of [15, Corol. 2], but use $(2.5)$ and $[15,(1.1) \Rightarrow(1.2)]$ in place of $[15,(1.3) \Rightarrow(1.2)]$.

In regard to (2.6.1), it should be noted that $Q^{\prime} \cap R$ may not be a prime divisor of $(b R)_{a}$. For example, let $(R, Q)$ be as in [8, Example 2, pp. 203-205] in the case $r=1$ and $m=0$, so altitude $R=2$ and there exists a height one maximal ideal $Q^{\prime}$ in $R^{\prime}$. Let $x$ be as in [8] and let $b=x^{2}-x$. Then $(b R)_{a}=(b, b x) R$ is the height one prime ideal $(x-1) R^{\prime} \cap R$, so $Q$ is not a prime divisor of $(b R)_{a}$, even though $Q^{\prime}$ is a prime divisor of $b R^{\prime}$.

It was shown in (2.4) that the sets Ass $\left(R /\left(I^{l}\right)_{a}\right)$ are increasing. They are also eventually all equal, as is shown in (2.7). (2.7) is a considerable extension of the first two referenced results, and it also extends the third referenced result from the Noetherian domain case.

(2.7) Theorem. (Cf. [5, Props. 7 and 18], [14, (2.5)], and [15, Corol. 3].) If $I$ is an ideal in a Noetherian ring $R$, then the sets $\operatorname{Ass}\left(R /\left(I^{i}\right)_{a}\right)$ are equal for all large $i$. In fact, for all large $i$,

$$
\begin{gathered}
\operatorname{Ass}\left(R /\left(I^{i}\right)_{a}\right)=\left\{p^{\prime} \cap R ; p^{\prime} \text { is a (height one) prime divisor of } u \Re(R, I)^{\prime}\right\} \\
=\left\{P \cap R ; P \text { is a prime divisor of }\left(u^{n} \mathfrak{R}\right)_{a} \text { for some } n \geq 1\right\} .
\end{gathered}
$$

Proof. Let $\Re=\Re(R, I)$. Then $u^{i} \Re^{\prime} \cap R=\left(I^{i}\right)_{a}$ for all $i \geq 1$. Also, since $u$ is a regular element, the prime divisors of $u^{i} \Re^{\prime}$ are the prime divisors of $u \Re^{\prime}$. Therefore

$$
\operatorname{Ass}\left(R /\left(I^{i}\right)_{a}\right) \subseteq\left\{p^{\prime} \cap R ; p^{\prime}\right. \text { is a (height one) }
$$

prime divisor of $\left.u \Re^{\prime}\right\}$,

and since $\Re^{\prime}$ is the integral closure of a Noetherian ring, $u \Re^{\prime}$ has only finitely many prime divisors. Therefore the sets $\operatorname{Ass}\left(R /\left(I^{l}\right)_{a}\right)$ are constant for all large $i$, by (2.4), and are contained in $\left\{p^{\prime} \cap R ; p^{\prime}\right.$ is a prime divisor of $\left.u \Re^{\prime}\right\}$.

Now if $p^{\prime}$ is a prime divisor of $u \Re^{\prime}$, then $p^{\prime} \cap \Omega$ is a prime divisor of $\left(u^{l} \Re\right)_{a}$ for all large $i$, by (2.6.1). Also, if $P$ is a prime divisor of $\left(u^{l} \Re\right)_{a}$ for 
some $i \geq 1$, then there exists a prime divisor $p^{\prime \prime}$ of $u^{i} \Re^{\prime}$ (and hence of $\left.u \Re^{\prime}\right)$ that lies over $P$, since $u^{i} \Re^{\prime} \cap \Re=\left(u^{i} \Re\right)_{a}$. Therefore

$$
\begin{aligned}
\{P ; P \text { is a prime divisor of } & \left.\left(u^{i} \Re\right)_{a} \text { for some } i \geq 1\right\} \\
& =\left\{p^{\prime} \cap \Re ; p^{\prime} \text { is a prime divisor of } u \Re^{\prime}\right\} .
\end{aligned}
$$

Finally, if $p^{\prime}$ is a prime divisor of $u \Re^{\prime}$, then either height $p^{\prime} \cap R=0$, and so clearly $p^{\prime} \cap R$ is a prime divisor of $\left(I^{i}\right)_{a}$ for all $i \geq 1$, or height $p^{\prime} \cap R>0$ and $t I \not P=p^{\prime} \cap \Re$, by (2.2). Also, $P$ is a prime divisor of $\left(u^{i} \Re\right)_{a}$ for some $i \geq 1$, by (2.6.1), so $\left(u^{i} \Re\right)_{a}: b t^{k} \Re=P$ for some homogeneous element $b t^{k}$ in $R$, since $\left(u^{i} \Re\right)_{a}$ is homogeneous. Then with $c t \in R-P$, it follows as in the proof of (2.4) that $p^{\prime} \cap R=P \cap R$ is a prime divisor of $\left(I^{i+k+j}\right)_{a}$ for all $j \geq 0$. Therefore, by the preceding two paragraphs,

$$
\begin{aligned}
& \operatorname{Ass}\left(R /\left(I^{\imath}\right)_{a}\right)=\left\{p^{\prime} \cap R ; p^{\prime} \text { is a prime divisor of } u \Re^{\prime}\right\} \\
& \quad=\left\{P \cap R ; P \text { is a prime divisor of }\left(u^{n} \Re\right)_{a} \text { for some } n \geq 1\right\} .
\end{aligned}
$$

This section will be closed with the following extension of [14, (2.6.1)] to all ideals in all Noetherian rings.

(2.8) Theorem. $(C f .[14,(2.6 .1)]$.$) If I is an ideal in a Noetherian ring$ $R$, then $\hat{A}^{*}(I) \subseteq A^{*}(I)$.

Proof. Let $Q \in \hat{A}^{*}(I)$. If height $Q=0$, then clearly $Q \in A^{*}(I)$. If height $Q \geq 1$, then there exists a prime divisor $p^{\prime}$ of $u \Re^{\prime}$ such that $t I \nsubseteq p^{\prime}$ and $p^{\prime} \cap R=Q$, by (2.7) and (2.2), where $\Re=\Re(R, I)$. Let $P=p^{\prime} \cap \Re$. Then $P$ is a prime divisor of $u \Re$, by [12, Thm. 2.15], and $t I \not \subset$. Therefore $P=u \Re: b t^{k} \Re$ for some homogeneous element $b t^{k} \in \mathcal{R}$, and $c t \notin P$ for some $c \in I$. Therefore

$$
P=u^{k+j+1} \Re: b c^{j} \Re=u \Re: b t^{k} c^{j} t^{j} \Re,
$$

so contracting to $R$ it follows that $I^{k+j+1}: b c^{j} R=Q$ for all $j \geq 0$, so $Q \in A^{*}(I)$.

3. On the monotonicity of $\operatorname{Ass}\left(R / I^{i}\right)$. It was shown in (2.4) that the sets $\operatorname{Ass}\left(R /\left(I^{i}\right)_{a}\right)$ are increasing, and it was shown in [1] that this does not hold for the sets $\operatorname{Ass}\left(R / I^{i}\right)$. However, the following is still an open problem:

(3.1) Question. If $I$ is an ideal in a Noetherian ring $R$ and $Q \in A^{*}(I)$ is a prime divisor of $I^{i}$ for some $i \geq 1$, is $Q \in \operatorname{Ass}\left(R / I^{n}\right)$ for all $n \geq i$ ? 
Of course, (3.1) holds for ideals of the form $I=J^{i}$ with $J$ an ideal and $i$ large, by the existence and definition of $A^{*}(J)$. (3.2) gives several equivalent sufficient conditions for (3.1) to hold for a given ideal $I$.

(3.2) THEOREM. Let I be an ideal in a Noetherian ring $R$ and assume any of the following equivalent conditions hold:

(3.2.1) $I^{i+1}: I=I^{i}$ for all $i \geq 1$.

(3.2.2) There exists an integer $k$ and an element $b \in I^{k}$ such that $I^{k+i}: b R=I^{i}$ for all $i \geq 1$.

(3.2.3) $I^{i+1}=I^{i} \cap\left(I^{i+1}: Q\right) \cap\left(I^{i+2}: I\right)$ for all $i \geq 0$ and for all $Q \in \operatorname{Spec} R$ that contain $I$.

Then if $Q$ is a prime divisor of $I^{i}$ for some $i \geq 1$, then $Q$ is a prime divisor of $I^{n}$ for all $n \geq i$.

Proof. It is shown in $[14,(3.6)]$ that (3.2.1)-(3.2.3) are equivalent and each is equivalent to: every prime divisor of $u \Re(R, I)$ is relevant. Therefore the proof of this theorem is essentially the same as the proof of (2.4).

The following corollary is well known, but it is included here because it follows quite readily from (3.2).

(3.3) CORollary. ( $C f$. [19, Thm. 2.3].) Let $b_{1}, \ldots, b_{g}$ be an $R$-sequence in a Noetherian ring $R$ and let $I=\left(b_{1}, \ldots, b_{g}\right) R$. If $Q$ is a prime divisor of $I^{i}$ for some $i \geq 1$, then $Q$ is a prime divisor of $I^{n}$ for all $n \geq 1$.

Proof. In [19, Thm. 2.3] it is shown that if $Q \in \operatorname{Ass}\left(R / I^{i}\right)$ for some $i \geq 1$, then grade $Q=g$. Therefore, since the images of $b_{1}, \ldots, b_{g}$ are an $R_{Q}$-sequence and $Q_{Q}$ is a prime divisor of $I^{i} R_{Q}$, it follows that grade $Q_{Q}$ $=g$. Therefore $Q_{Q}$ must be a prime divisor of $I_{Q}$, so $Q$ is a prime divisor of $I$. Finally, it is well known that $I^{i+1}: b_{1} R=I^{i}$ for all $i \geq 1$, so it follows from (3.2) that $Q$ is a prime divisor of $I^{n}$ for all $n \geq 1$.

An important special case of (3.3) is considered in (3.4). For (3.4) recall that an ideal $I$ is of principal class in case $I$ can be generated by $h=$ height $I$ elements.

(3.4) Corollary. (Cf. [24, Lemma 5, p. 401].) Let I be an ideal of principal class in a Cohen-Macaulay ring $R$, say height $I=h$. Then every prime divisor of $I^{i}$ has height $h$ for all $i \geq 1$. 
Proof. $R$ satisfies the Unmixedness Theorem, by [8, (25.6)], so every prime divisor of $I$ has height $h$. Also, ideals of the principal class in $R$ are generated by $R$-sequences, since $R$ is Cohen-Macaulay, so the conclusion follows from (3.3).

This section will be closed with the following result which is closely related to (3.2) and which gives a sufficient condition for (3.1) to hold for a specified $Q \in A^{*}(I)$.

(3.5) Proposition. Let $I$ be an ideal in a Noetherian ring $R$ and let $Q \in \operatorname{Ass}\left(R / I^{i}\right)$ for some $i \geq 1$. If $I^{j+1}=I^{j} \cap\left(I^{j+1}: Q\right) \cap\left(I^{j+2}: I\right)$ for all $j \geq 0$, then $Q$ is a prime divisor of $I^{n}$ for all $n \geq i$.

Proof. By [14, (3.3)], the hypothesis implies $(Q R[t, u] \cap \Re, u) \Re$ $(=(u, Q, t I) \Re)$ is not contained in any (necessarily irrelevant) prime divisor of $u \Re$, so it follows that no irrelevant prime divisor of $u \Re$ lies over $Q$. Therefore the proof is essentially the same as the proof of (2.4).

4. A characterization of Cohen-Macaulay rings in terms of $\hat{A}^{*}(I)=$ $A^{*}(I)$. During the summer of 1980 , Steve McAdam and I both spent a few days investigating what we considered a very nice generalization of $R$-sequences (see the condition in (4.1)). After we had proved a few results about such sequences, McAdam's student, Dan Katz, showed us the result in (4.1), and so our new sequences were, in fact, just $R$-sequences. But at least we had found a new (to us) equivalence of the dfinition of an $R$-sequence, and this new equivalence is fairly useful, as is shown by the remaining results in this section.

(4.1) Lemma. Let $b_{1}, \ldots, b_{g}$ be elements in a Noetherian ring $R$, let $I_{J}=\left(b_{1}, \ldots, b_{j}\right) R$ for $j=0,1, \ldots, g$, and assume $I_{g} \neq R$. Then $b_{1}, \ldots, b_{g}$ are an $R$-sequence if and only if $I_{j-1}^{i}: b_{j} R=I_{j-1}^{i}$ for $j=1, \ldots, g$ and for all large $i$.

Proof. The necessity of the condition follows immediately from the definition of an $R$-sequence and (3.3), so assume the condition holds. Then it follows that $b_{1}$ is a regular element, so $b_{1}$ is an $R$-sequence. Therefore $b_{1} R$ and $b_{1}^{i} R$ have the same prime divisors for all $i \geq 1$, by (3.3), so the condition implies $b_{1} R: b_{2} R=b_{1} R$, hence $b_{1}, b_{2}$ are an $R$-sequence. Therefore the condition together with (3.3) imply $b_{1}, b_{2}, b_{3}$ are an $R$-sequence, and a repetition of this shows that $b_{1}, \ldots, b_{g}$ are an $R$-sequence. 
(4.1) yields an easy proof of the following characterization of CohenMacaulay rings.

(4.2) Proposition. A Noetherian ring $R$ is Cohen-Macaulay if and only if for all ideals $I$ of the principal class in $R$ it holds that $I^{\prime}$ has no imbedded prime divisors for all large $i$.

Proof. If $R$ is Cohen-Macaulay, then the condition holds by (3.4). Conversely, let $M$ be a maximal ideal in $R$, say height $M=h>0$, and let $b_{1}, \ldots, b_{h}$ in $M$ such that height $I_{j}=j$, where $I_{j}=\left(b_{1}, \ldots, b_{j}\right) R(j=$ $0,1, \ldots, h)$. Then $b_{J}$ is not in any minimal prime divisor of $I_{j-1}$, so $I_{j-1}^{l}: b_{j} R=I_{j-1}^{l}$ for all large $i$, by hypothesis. Therefore $b_{1}, \ldots, b_{h}$ are an $R$-sequence, by (4.1), so their images in $R_{M}$ are an $R_{M}$-sequence, hence $R_{M}$ is Cohen-Macaulay, and so it follows that $R$ is Cohen-Macaulay.

In 1980 in [15, Thm. 4] $I$ essentially characterized Noetherian rings $R$ such that $\hat{A}^{*}(I)=A^{*}(I)$ for all ideals $I$ in $R$. Since this is a useful property, it is somewhat disappointing that this class of such rings is quite small (for example, if altitude $R \geq 4$, then $R$ is not in this class). However, just asking that this property hold for certain types of ideals, such as ideals of the principal class, leads to more interesting classes. (4.3) shows that this property can be used to characterize Cohen-Macaulay rings. For (4.3), recall that a Noetherian ring $R$ is locally quasi-unmixed in case for each $P \in$ Spec $R$ all minimal prime ideals in $\left(R_{P}\right)^{*}$ have the same depth.

(4.3) TheOREM. Let $R$ be a locally quasi-unmixed Noetherian ring. Then $R$ is Cohen-Macaulay if and only if $\hat{A}^{*}(I)=A^{*}(I)$ for all ideals $I$ of the principal class in $R$.

Proof. If $R$ is a Cohen-Macaulay ring and $I$ is an ideal of the principal class in $R$, then $I^{i}$ has no imbedded prime divisors for all $i \geq 1$, by (3.4). Therefore $A^{*}(I) \subseteq \hat{A}^{*}(I)$, since it is clear that minimal prime divisors of $I$ are in $\hat{A}^{*}(I) \cap A^{*}(I)$. However, it is always true that $\hat{A}^{*}(I) \subseteq A^{*}(I)$, by (2.8), so $\hat{A}^{*}(I)=A^{*}(I)$.

Conversely, let $I$ be an ideal of the principal class in $R$. Then $\left(I^{l}\right)_{a}$ has no imbedded prime divisors for all $i \geq 1$, by [13, Thm. 2.12], since $R$ is locally quasi-unmixed, so $I^{l}$ has no imbedded prime divisors for all large $i$, by hypothesis, and so $R$ is Cohen-Macaulay, by (4.2).

Concerning (4.3), it should be noted that the quasi-unmixed assumption is necessary. For example, let $(R, M)$ be as in [8, Example 2, pp. 
203-205] in the case $m=0$ and $r=1$. Then $R$ is a local domain of altitude two and $\hat{A}^{*}(I)=A^{*}(I)$ for all ideals $I$ in $R$, by [15, (4.2.2)]. But since there exist a height one maximal ideal in $R^{\prime}, M \in \hat{A}^{*}(b R)=A^{*}(b R)$ for each nonzero $b$ in $M$, by (2.6.1), hence $R$ is not Cohen-Macaulay.

Even though the condition in (4.1) was not a generalization of an $R$-sequence, the same condition with $\left(I_{j-1}^{l}\right)_{a}$ replacing $I_{j-1}^{i}$ is a very useful generalization. Specifically:

(4.4) Definition. Let $b_{1}, \ldots, b_{g}$ be elements in a Noetherian ring $R$ and let $I_{j}=\left(b_{1}, \ldots, b_{j}\right) R(j=0,1, \ldots, g)$. Then $b_{1}, \ldots, b_{g}$ are an asymptotic sequence in case $I_{g} \neq R$ and $\left(I_{j-1}^{i}\right)_{a}: b_{j} R=\left(I_{j-1}^{i}\right)_{a}$ for $j=1, \ldots, g$ and for all large $i$.

It is shown in [17] that most of the basic properties of $R$-sequences have valid analogues for asymptotic sequences. It is also shown in [17, (2.3.5)] that an $R$-sequence is an asymptotic sequence, but not conversely. (4.5) gives a sufficient condition for the converse to hold.

(4.5) Proposition. Let $R$ be a Noetherian ring such that $\hat{A}^{*}(I)=A^{*}(I)$ for all ideals $I$ of the principal class. Then elements $b_{1}, \ldots, b_{g}$ in $R$ are an $R$-sequence if and only if they are an asymptotic sequence.

Proof. Let $I_{j}=\left(b_{1}, \ldots, b_{j}\right) R(j=0,1, \ldots, g)$. If $b_{1}, \ldots, b_{g}$ are an $R$-sequence, then for $j=1, \ldots, g$ it holds that $b_{j} \notin \cup\left\{Q ; Q \in A^{*}\left(I_{j-1}\right)\right\}=$ $\cup\left\{Q ; Q \in \hat{A}^{*}\left(I_{j-1}\right)\right\}$, by (3.3) and hypothesis, so $b_{1}, \ldots, b_{g}$ are an asymptotic sequence, by (4.4).

Conversely, $b_{j} \notin \cup\left\{Q ; Q \in \hat{A}^{*}\left(I_{J-1}\right)\right\}=\cup\left\{Q ; Q \in A^{*}\left(I_{j-1}\right)\right\}$, by definition and hypothesis (it follows immediately from (4.4) that an ideal generated by an asymptotic sequence is an ideal of the principal class). Therefore $I_{j-1}^{i}: b_{J} R=I_{J-1}^{i}$ for $j=1, \ldots, g$ and for all large $i$, so $b_{1}, \ldots, b_{g}$ are an $R$-sequence, by (4.1).

This section will be closed with the following important special case of (4.5).

(4.6) Corollary. Elements $b_{1}, \ldots, b_{g}$ in a Cohen-Macaulay ring $R$ are an $R$-sequence if and only if they are an asymptotic sequence.

Proof. $\hat{A}^{*}(I)=A^{*}(I)$ for all ideals $I$ of the principal class in $R$, by the first paragraph of the proof of (4.3), so the conclusion follows from (4.5). 
5. A characterization of an altitude two local UFD in terms of $\hat{A}^{*}(I)$. In 1979 in [5, Prop. 24] it was shown that if $R$ is a Noetherian domain of altitude two which is locally a UFD, then $\operatorname{Ass}\left(R / I^{i}\right)=\operatorname{Ass}\left(R /\left(I^{j}\right)_{a}\right)$ for all ideals $I$ in $R$ and for all positive integers $i$ and $j$. In this brief section it is shown that this condition characterizes local UFDs of altitude two.

(5.1) THEOREM. Let $(R, M)$ be a Noetherian domain such that altitude $R \geq 2$. Then altitude $R=2$ and $R$ is a UFD if and only if $\operatorname{Ass}\left(R / I^{i}\right)=$ $\operatorname{Ass}\left(R /\left(I^{J}\right)_{a}\right)$ for all ideals $I$ in $R$ and for all positive integers $i$ and $j$.

Proof. The necessity of the condition was proved in [5, Prop. 24], so assume the condition holds. Assume first that height $M=2$. Now it is clear that $\hat{A}^{*}(I)=A^{*}(I)$ for all ideals $I$ in $R$, so either $R=R^{\prime}$ or there exists a height one maximal ideal in $R^{\prime}$, by [15, (4.2.2)]. Thus if $R \neq R^{\prime}$, then $M \in \hat{A}^{*}(I)$ for all nonzero ideals $I$ in $R$, by (2.6), so if $p$ is a height one prime ideal in $R$, then $M \in \hat{A}^{*}(p)$. However, this and the condition imply $M$ is a prime divisor of $p$, so it follows that $R=R^{\prime}$.

Let $p$ be a height one prime ideal in $R$ and suppose $p$ is not principal. Then $M \in \hat{A}^{*}(p)$, by [5, Prop 21], and this and the condition then imply the contradiction that $M$ is a prime divisor of $p$. Therefore, if altitude $R=2, R$ is a UFD.

Now suppose altitude $R>2$. Then, by [15, Thm. 4], altitude $R=3$ and either: (a) $R=R^{\prime}$ and $R$ is not quasi-unmixed; or, (b) there exists a height one maximal ideal in $R^{\prime}$. If (a) holds, there exists a minimal prime ideal $z$ in $R^{*}$ such that depth $z<3$. Since $R=R^{\prime}$, depth $z>1$, by [15, $(1.1) \Rightarrow(1.3)]$, so depth $z=2$. Then there exist at most finitely many $P^{*} \in \operatorname{Spec} R^{*}$ such that $z \subset P^{*}$ and height $P^{*}=2$, by [4, Thm. 1]. Therefore there exists a height two prime ideal $P$ in $R$ such that $z+P R^{*}$ is $M^{*}$-primary, so $M \in \hat{A}^{*}(P)$, by (6.6). But this and the condition lead to a contradiction. Therefore (b) holds. However, this implies $M \in \hat{A}^{*}(I)$ for all nonzero ideals $I$ in $R$, by (2.6), and this and the condition again lead to a contradiction. Therefore $R$ is an altitude two UFD.

(5.2) RemarK. (5.2.1) Since $\hat{A}^{*}(I) \subseteq A^{*}(I)$ always holds, by (2.8), it follows from the proof of (5.1) that the following conditions are equivalent for a local domain $R$ such that altitude $R \geq 2$ : (a) Altitude $R=2$ and $R$ is a UFD; (b) for each ideal $I$ in $R$ the sets $\operatorname{Ass}\left(R / I^{i}\right)$ are equal for all $i \geq 1$; and, (c) for each ideal $I$ in $R$ the sets $\operatorname{Ass}\left(R /\left(I^{i}\right)_{a}\right)$ are equal for all $i \geq 1$. 
(5.2.2) It follows immediately from (5.1) and [5, Prop. 24] that a Noetherian domain which is not a field satisfies the condition in (5.1) if and only if for each maximal ideal $M$ in $R$, either height $M=1$ or $R_{M}$ is an altitude two UFD.

6. $\hat{A}^{*}(I)$ in flat extension rings and certain factor rings. In this section it is shown in (6.3) that if $I$ is an ideal in a Noetherian ring $R$, then the sets $\hat{A}^{*}\left((I+z) / z\right.$ ) (with $z \in \mathrm{mAss} R$ ) are known once $\hat{A}^{*}(I)$ is known, and conversely. And in (6.9) and (6.5) it is shown that if $S$ is a Noetherian ring which is a faithfully flat $R$-module, then $\hat{A}^{*}(I)$ is known when $\hat{A}^{*}(I S)$ is, and knowledge about $\hat{A}^{*}(I)$ provides considerable information about $\hat{A}^{*}(I S)$. These results are quite technical, but they are also very useful, as is shown in [17].

We begin with two lemmas, the first of which is closely related to (2.6.1).

(6.1) LemMa. Let $R$ be a Noetherian ring, let $A$ be a ring between $R$ and $R^{\prime}$, and let $b$ be a regular element in $A$. Then if $P \in \operatorname{Ass}\left(A /\left(b^{i}\right)_{a}\right)$ for some $i \geq 1$, there exists a height one prime divisor $p^{\prime}$ of $b R^{\prime}$ that lies over $P$. And, if $p^{\prime \prime}$ is a prime divisor of $b R^{\prime}$, then $p^{\prime \prime} \cap A$ is a prime divisor of $\left(b^{i} A\right)_{a}$ for all large $i$.

Proof. Since $\left(b^{i} A\right)_{a}=b^{i} R^{\prime} \cap A$, the first statement is clear. To complete the proof let $p^{\prime \prime}$ be a prime divisor of $b R^{\prime}$ and note that there exists a finite extension ring $B$ of $R$ such that $B \subseteq A, b \in B$, and $p^{\prime \prime} \cap A$ is the only prime ideal in $A$ that lies over $p^{\prime \prime} \cap B$. Therefore, since $\left(b^{i} B\right)_{a}=b^{i} R^{\prime}$ $\cap B=b^{i} R^{\prime} \cap A \cap B=\left(b^{i} A\right)_{a} \cap B$ and $p^{\prime \prime} \cap B \in \hat{A}^{*}(b B)$, by (2.6.1), it follows that $p^{\prime \prime} \cap A$ is a prime divisor of $\left(b^{i} A\right)_{a}$ for all large $i$.

(6.2) LEMMA. Let $R$ be a Noetherian ring, let $A$ be a ring between $R$ and $R^{\prime}$, let $b$ be a regular element in $A$, such that $b A=(b A)_{a}$, let $z \in \mathrm{mAss} A$, and let ${ }^{-}$denote residue class modulo $z$. Then there is a one-to-one correspondence between the sets $\{P ; P$ is a prime divisor of $b A$ and $z \subset P\}$, $\left\{p ; p\right.$ is a prime divisor of $\left(\bar{b}^{i} \bar{A}\right)_{a}$ for some $\left.i \geq 1\right\}$, and $\{Q ; Q$ is a prime divisor of $\left.\bar{b} \overline{A^{\prime}}\right\}$ given by $A_{P} / z A_{P}=\bar{A}_{p}=\left(\overline{A^{\prime}}\right)_{Q}$, and these rings are discrete valuation rings.

Proof. Let $Z=\operatorname{Rad} A$, so $Z \subseteq b A=(b A)_{a}$, so there exists a one-toone correspondence between the prime divisors of $b A$ and of $(b A) / Z$ such that $A_{P} / Z A_{P}=(A / Z)_{P / Z}$. (By [12, Corol. 2.11], $b A$ is a finite intersection of height one primary ideals.) Also, $(b A) / Z=((b A) / Z)_{a}$, so there exists 
a one-to-one correspondence between the prime divisors $P / Z$ of $(b A) / Z$ and the prime divisors $P^{\prime}$ of $(b+Z)(A / Z)^{\prime}$ given by $(A / Z)_{P / Z}=$ $\left((A / Z)^{\prime}\right)_{P^{\prime}}$ and these rings are discrete valuation rings, by [12, Corol. 2.11, Prop. 2.7, and Thm. 2.15]. Let $z^{\prime}$ be the minimal prime ideal in $(A / Z)^{\prime}$ that lies over $z / Z$. Then $\overline{A^{\prime}}=(A / Z)^{\prime} / z^{\prime}$ is a direct summand of $(A / Z)^{\prime}$, so the prime divisors of $\bar{b} \overline{A^{\prime}}$ are the ideals $P^{\prime} / z^{\prime}$ with $P^{\prime}$ a prime divisor of $(b+Z)(A / Z)^{\prime}$ that contains $z^{\prime}$, and these lie over the ideals $\bar{P}$ in $\bar{A}$ with $P$ a prime divisor of $b A$ that contains $z$, by the one-to-one correspondence. But $\left(\bar{b}^{l} \bar{A}\right)_{a}=\bar{b}^{l} \bar{A}^{\prime} \cap \bar{A}$, so the conclusion follows from (6.1).

The first of the main results in this section shows the nice behavior of $\hat{A}^{*}(I)$ between $R$ and the rings $R / z$ with $z \in$ mAss $R$.

(6.3) THEOREM. The following statements hold for an ideal $I$ in a Noetherian ring $R$ :

(6.3.1) If $P \in \hat{A}^{*}(I)$, there exists $z \in \mathrm{mAss} R$ such that $z \subseteq P$ and $P / z \in \hat{A}^{*}((I+z) / z)$.

(6.3.2) if $z \in \mathrm{mAss} R$ and $Q \in \hat{A}^{*}((I+z) / z)$, there exists $P \in \hat{A}^{*}(I)$ such that $z \subseteq P$ and $P / z=Q$. Moreover, $P \in \hat{A}^{*}(I+z)$.

Proof. (6.3.1) Let $P \in \hat{A}^{*}(I)$ and let $\Re=\mathscr{R}(R, I)$. Then there exists a (height one) prime divisor $p^{\prime}$ of $u R^{\prime}$ such that $p^{\prime} \cap R=P$, by (2.7). Let $z^{\prime}$ be the minimal prime ideal in $\Re^{\prime}$ which is contained in $p^{\prime}$ (see [12, Props. 2.13 and 2.7]) and let $z=z^{\prime} \cap R$. Then $z \subseteq P, z \in \mathrm{mAss} R$, and $z^{\prime}=z T \cap \Re^{\prime}$, where $T$ is the total quotient of $R$. Also, $R / z \subseteq$ $\Re /\left(z^{\prime} \cap \Re\right) \subseteq \Re^{\prime} / z^{\prime} \subseteq\left(\Re /\left(z^{\prime} \cap \Re\right)\right)^{\prime}$ and $p^{\prime} / z^{\prime}$ is a height one prime ideal in $\Omega^{\prime} / z^{\prime}$ that lies over $P / z$, so there exists a height one prime divisor of $u+z^{\prime}$ in $\left(\Re^{\prime} / z^{\prime}\right)^{\prime}$ that lies over $p^{\prime} / z^{\prime}$. Therefore, since $\Re /\left(z^{\prime} \cap \Re\right) \cong \Re(R / z,(I+z) / z)=($ say $) \Re_{0}$, by [20, Lemma 1.1], there exists a height one prime divisor of $u\left(\Omega_{0}\right)^{\prime}$ that lies over $P / z$, hence $P / z \in \hat{A}^{*}((I+z) / z)$, by $(2.7)$.

(6.3.2) Let $Q \in \hat{A}^{*}((I+z) / z)$, let $\Re, \Omega_{0}$, and $T$ be as in the preceding paragraph, and let $z^{*}=z T \cap \Re^{\prime}$, so $\mathcal{R} /\left(z^{*} \cap \Re\right) \cong \mathcal{R}_{0}$. Since $Q \in \hat{A}^{*}((I+z) / z)$, there exists a height one prime divisor $q^{\prime}$ of $u \Omega_{0}^{\prime}$ that lies over $Q$, by (2.7). Let $\delta$ be the ring such that $\Re_{0} \subseteq \delta \subseteq \Re_{0}^{\prime}$ and $\delta \cong \Re^{\prime} / z^{*}$ and let $q=q^{\prime} \cap \mathcal{\delta}$, so $q$ is a prime divisor of $\left(u^{i} \delta\right)_{a}$ for some $i \geq 1$, by (6.1). Let $p^{\prime} \in \operatorname{Spec} \Re^{\prime}$ such that $z^{*} \subset p^{\prime}$ and $p^{\prime} / z^{*}$ corresponds to $q$. Then $p^{\prime} / z^{*}$ is a prime divisor of $\left(\left(u^{i}+z^{*}\right)\left(\Re^{\prime} / z^{*}\right)\right)_{a}$, by the isomorphism, so (6.2) implies $p^{\prime}$ is a (height one) prime divisor of $u \Re^{\prime}$. Let $P=p^{\prime} \cap \Re$. Then $z \subseteq P$ and $P / z=Q$, since $q^{\prime} \cap(R / z)=Q$, and 
$P \in \hat{A}^{*}(I)$, by (2.7). Finally, applying what was just shown to the ideal $I+z$ in place of $I$, it follows quite readily (since $\mathcal{R}^{\prime} \subseteq \mathbb{Q}^{\prime}=\mathscr{R}(R, I+z)^{\prime}$ and $\left.\mathscr{R}^{\prime} / z^{*} \cong \mathbb{Q}^{\prime} /\left(z T \cap \mathbb{Q}^{\prime}\right)\right)$ that $P \in \hat{A}^{*}(I+z)$.

To prove the second of the main results in this section we need the following lemma. (6.4) is known, but I know no reference for it, so it is given here together with a brief sketch of its proof.

(6.4) Lemma. Let $R \subseteq S$ be Noetherian rings such that $S$ is a faithfully flat $R$-module, let $X_{1}, \ldots, X_{n}$ be indeterminates, and let $f_{1}, \ldots, f_{k}$ be elements in the total quotient ring of $R\left[X_{1}, \ldots, X_{n}\right]$. Then $S\left[X_{1}, \ldots, X_{n}, f_{1}, \ldots, f_{k}\right]$ is a faithfully flat $R\left[X_{1}, \ldots, X_{n}, f_{1}, \ldots, f_{k}\right]$-module.

Proof. $B^{*}=S\left[X_{1}, \ldots, X_{n+k}\right]$ is a faithfully flat $B=R\left[X_{1}, \ldots, X_{n+k}\right]-$ module. Let $K^{*}$ and $K$ be the kernels of the natural homomorphisms from $B^{*}$ onto $S\left[X_{1}, \ldots, X_{n}, f_{1}, \ldots, f_{k}\right]$ and from $B$ onto $R\left[X_{1}, \ldots, X_{n}, f_{1}, \ldots, f_{k}\right]$, respectively. Then it is quite straightforward to see that $K^{*}=K B^{*}$, since the $f_{i}$ are in the total quotient ring of $R\left[X_{1}, \ldots, X_{n}\right]$ and since $S\left[X_{1}, \ldots, X_{n}\right]$ is a faithfully flat $R\left[X_{1}, \ldots, X_{n}\right]$-module, and the conclusion clearly follows from this.

(6.5) and (6.8) consider the relationship between $\hat{A}^{*}(I)$ and $\hat{A}^{*}(I S)$, where $I$ is an ideal in a Noetherian ring $R$ and $S$ is a flat $R$-algebra.

(6.5) Theorem. Let $R \subseteq S$ be Noetherian rings such that $S$ is a flat $R$-module, let $I$ be an ideal in $R$, let $P \in \hat{A}^{*}(I)$, and let $P^{*}$ be a minimal prime divisor of $P S$. Then $P^{*} \in \hat{A}^{*}(I S)$.

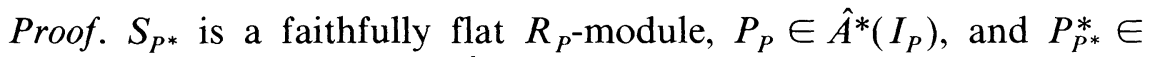
$\hat{A}^{*}\left(I S_{P^{*}}\right)$ if and only if $P^{*} \in \hat{A}^{*}(I S)$. Therefore it may be assumed to begin with that $R$ and $S$ are local with maximal ideals $P$ and $P^{*}$, respectively, and that $P S$ is $P^{*}$-primary. Let $\Re=\Re(R, I)$ and $\delta=$ $\mathcal{R}(S, I S)$, and by (2.7) and the hypothesis let $p^{\prime}$ be a height one prime divisor of $u \Re^{\prime}$ that lies over $P$. Let $f \in p^{\prime}$ such that $f$ is not in any other prime ideal in $\Re^{\prime}$ that lies over $p^{\prime} \cap \Re$, so $p^{\prime} \cap \Re[f]$ is a height one prime divisor of $u \Re[f]$. Now $\Re[f] \subseteq \delta[f]$ are Noetherian rings such that $\delta[f]$ is a faithfully flat $\Re[f]$-module, by (6.4), and $\delta[f] \subseteq \mathcal{S}^{\prime}$, since $f \in R^{\prime} \subseteq \mathcal{S}^{\prime}$. Let $p^{*}$ be a minimal prime divisor of $\left(p^{\prime} \cap \Re[f]\right) \delta[f]$. Then height $p^{*}=1$ and $p^{*}$ is a prime divisor of $u \delta[f]$, so there exists a height one prime divisor of $u S^{\prime}$ that lies over $p^{*}$. Hence, since $p \cap R=$ $P$ and $P S$ is $P^{*}$-primary, it follows from (2.7) that $P^{*}=p^{*} \cap S \in$ $\hat{A}^{*}(I S)$. 
To prove the last of the main results in this section we need one further lemma. This lemma was proved in [17, (2.4)], but it is included here for the sake of completeness.

(6.6) LemMa. Let $R$ and $S$ be Noetherian rings such that $S$ is a flat $R$-module and let $P$ be a minimal prime divisor of $(I, z) S$, where $I$ is an ideal in $R$ and $z \in \mathrm{mAss} S$. Then $P \in \hat{A}^{*}(I S), \varphi^{-1}(P) \in \hat{A}^{*}(I)$, and $P$ is a prime divisor of $\left(I^{i}\right)_{a} S$ for all large $i$, where $\varphi$ is the natural homomorphism from $R$ to $S$.

Proof. The proof is essentially the same as the proof of $[16,(2.9 .2)]$, where the lemma is proved under the assumption that $S_{P} /\left(\operatorname{Rad} S_{P}\right)$ is analytically unramified. This assumption was used in [16] to invoke a result in the proof of $\left[15\right.$, Thm. 1] to show that $\cap\left\{\left(P^{i} S_{P}\right)_{a} ; i \geq 1\right\}=$ $\operatorname{Rad} S_{P}$. However, it was shown in [3] that this holds in all Noetherian rings $S$, so use [3] in place of the result in [15, Thm. 1] in the proof given in [16].

The following corollary of (6.6) is often useful.

(6.7) COROllary. Let $R, S, I$, and $z$ be as in (6.6) and assume that a prime ideal $P$ in $S$ is a minimal prime divisor of $(I, z) S$. Then $P$ is a prime divisor of $\varphi^{-1}(P) S$.

Proof. By (6.6), $P$ is a prime divisor of $\left(I^{i}\right)_{a} S$, so $P$ is a prime divisor of $p S$ for some prime divisor $p$ of $\left(I^{i}\right)_{a}$, by $[8,(18.11)]$, and then necessarily $\varphi^{-1}(P)=p$.

(6.8) contains another useful result concerning $\hat{A}^{*}(I)$ and $\hat{A}^{*}(I S)$.

(6.8) THEOREM. Let $R \subseteq S$ be Noetherian rings such that $S$ is a flat $R$-module, let $I$ be an ideal in $R$, and let $P^{*} \in \hat{A}^{*}(I S)$. Then $P^{*} \cap R \in$ $\hat{A}^{*}(I)$.

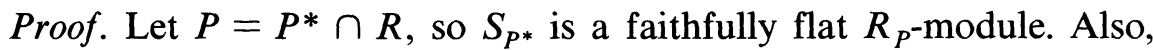
$P_{P^{*}}^{*} \in \hat{A}^{*}\left(I S_{P}\right)$, and $P \in \hat{A}^{*}(I)$ if and only if $P_{P} \in \hat{A}^{*}\left(I_{P}\right)$, so it may be assumed to begin with that $S$ is a faithfully flat $R$-module and that $P^{*}$ and $P$ are the unique maximal ideals in $S$ and $R$, respectively. Let $S^{*}$ be the completion of $S$, so $R \subseteq S^{*}$ satisfy the same conditions as $R \subseteq S$. Also, if $N$ is the maximal ideal in $S^{*}$, then $N \in \hat{A}^{*}\left(I S^{*}\right)$, by (6.5), so it may be assumed that $S$ is complete. Let $\Re=\mathscr{R}(R, I)$ and $\mathcal{S}=\mathscr{R}(S, I S)$, so $\Re \subseteq \mathcal{S}$ are Noetherian rings and $\mathcal{S}$ is a faithfully flat $\mathcal{R}$-module, by (6.4). 
By hypothesis and (2.7), there exists a height one prime divisor $p^{* \prime}$ of $u \delta^{\prime}$ that lies over $P^{*}$. Let $p^{*}=p^{* \prime} \cap \mathcal{S}$, and let $z$ be the minimal prime ideal in $\mathcal{S}^{\prime}$ that is contained in $p^{* \prime}$. Then $S /(z \cap S)$ is a complete local domain, since $S$ is complete, so by the altitude formula it follows that $\left(p^{* \prime} / z\right) \cap$ $(\mathcal{S} /(z \cap \mathcal{S}))$ is a height one prime ideal, hence $p^{*}$ is a minimal prime divisor of $(u, z \cap \mathcal{S}) \mathcal{S}$. Therefore, since $z \cap \mathcal{S}$ is a minimal prime ideal, $p=p^{*} \cap \Re \in \hat{A}^{*}(u \Re)$, by (6.6). Thus there exists a height one prime divisor of $u \Re^{\prime}$ that lies over $p$, since $u^{n} \Re^{\prime} \cap \Re=\left(u^{n} \Re\right)_{a}$ for all $n \geq 1$, and so $P=p \cap R \in \hat{A}^{*}(I)$, by (2.7).

This section will be closed with the following corollary of (6.5) and (6.8).

(6.9) Corollary. Let $R \subseteq S$ be Noetherian rings such that $S$ is a faithfully flat $R$-module and let $I$ be an ideal in $R$. Then $\hat{A}^{*}(I)=\left\{P^{*} \cap R\right.$; $\left.P^{*} \in \hat{A}^{*}(I S)\right\}$.

Proof. If $P \in \hat{A}^{*}(I)$, then $P S \neq S$ and each prime divisor of $P S$ lies over $P$, by faithful flatness, so the conclusion follows from (6.5) and (6.8).

7. On $\hat{A}^{*}(I)$ and $l(I)$. In this section several necessary and sufficient conditions are given for the maximal ideal in a local ring $R$ to be in $\hat{A}^{*}(I)$ for a given ideal $I$ in $R$. We begin by recalling the following definition.

(7.1) Definition. If $I$ is an ideal in a local ring $(R, M)$, then the analytic spread of $I$, denoted $l(I)$, is defined to be the depth of the ideal $(M, u) \Re$ in the ring $\Re=\mathscr{R}(R, I)$.

(7.2) contains a couple of useful results concerning $l(I)$.

(7.2) Lemma. Let $\left(R^{*}, M^{*}\right)$ be the completion of a local ring $(R, M)$ and let $I$ be an ideal in $R$. Then:

(7.2.1) $l(I) \geq l((I+w) / w)$ for all $w \in \mathrm{mAss} R$ and equality holds for some $w$.

(7.2.2) $l(I)=l\left(I R^{*}\right)$.

Proof. Let $\Re=\Re(R, I)$ and $\delta=\Re\left(R^{*}, I R^{*}\right)$. Then $\Re /(M, u) \Re=$ $\delta /\left(M^{*}, u\right) \mathcal{S}$, so $l(I)=l\left(I R^{*}\right)$. Let $w \in$ mAss $R$ and let $w^{\prime}=w R[t, u] \cap$ $\Re$, so $w^{\prime} \in \operatorname{mAss} \Re$ and $R / w^{\prime} \cong \Re(R / w,(I+w) / w)$, by [20, Lemma 1.1]. Therefore, if $P$ is a prime divisor of $(M / w, u)\left(\Re / w^{\prime}\right)$ such that 
depth $P=l((I+w) / w)$ and $Q$ is the preimage of $P$ in $\mathcal{R}$, then $(M, u) \mathscr{R}$ $\subseteq Q$, so

$$
l(I)=\operatorname{depth}(M, u) \Re \geq \operatorname{depth} Q=l((I+w) / w) .
$$

Finally, let $Q$ be a minimal prime divisor of $(M, u) \Re$ such that depth $Q=$ $l(I)$ and let $z^{\prime} \in \mathrm{mAss} R$ such that $z^{\prime} \subseteq Q$. Then $z=z^{\prime} \cap R \in \mathrm{mAss} R$ and $z^{\prime}=z R[t, u] \cap \mathscr{R}$, so $R / z^{\prime} \cong \Re(R / z,(I+z) / z)$ and $(M / z, u)\left(\Re / z^{\prime}\right) \subseteq Q / z^{\prime}$, so it follows that $l((I+z) / z)=l(I)$.

In [21, Lemma 1.1] it was shown that if $I$ is an ideal in a local ring $(R, M)$ and if $l(I)=$ altitude $R$, then there exists a height one prime divisor $P$ of $u \mathscr{R}(R, I)$ such that $P \cap R=M$, and that the converse holds when $R$ is quasi-unmixed. Actually, somewhat more can be said in this case, as is shown in (7.3).

(7.3) TheOREM. Let $(R, M)$ be a local ring, let $\left(R^{*}, M^{*}\right)$ be the completion of $R$, and let $I$ be an ideal in $R$. Then, for the following statements, (7.3.2)-(7.3.5) are equivalent and are implied by (7.3.1), and (7.3.1)-(7.3.5) are equivalent if $R$ is quasi-unmixed.

(7.3.1) $l(I)=$ altitude $R$.

(7.3.2) $M \in \hat{A}^{*}(I)$.

(7.3.3) $M^{*} \in \hat{A}^{*}(I R)$.

(7.3.4) There exists $z \in \mathrm{mAss} R^{*}$ such that $M^{*} / z \in \hat{A}^{*}\left(\left(I R^{*}+z\right) / z\right)$.

(7.3.5) There exists $z \in \mathrm{mAss} R^{*}$ such that $\operatorname{depth} z=l\left(\left(I R^{*}+z\right) / z\right)$.

Proof. Let $\Re=\mathscr{R}(R, I)$ and $\delta=\mathscr{R}\left(R^{*}, I R^{*}\right)$. Then, by $(7.2), l(I)=$ $l\left(I R^{*}\right)=l\left(\left(I R^{*}+z\right) / z\right)$ for some $z \in$ mAss $R^{*}$. Therefore, if (7.3.1) holds, then

$$
\text { altitude } R=l(I)=l\left(\left(I R^{*}+z\right) / z\right) \leq \text { altitude } R^{*} / z,
$$

by [2], and

altitude $R^{*} / z=$ depth $z \leq$ altitude $R^{*}$,

so (7.3.1) $\Rightarrow$ (7.3.5). Now $R^{*} / z$ satisfies the altitude formula, so $M^{*} / z \in$ $\hat{A}^{*}\left(\left(I R^{*}+z\right) / z\right)$ if and only if $l\left(\left(I R^{*}+z\right) / z\right)=\operatorname{depth} z$, by [6, Thm. 3], so (7.3.5) $\Leftrightarrow$ (7.3.4). Also, (7.3.4) $\Leftrightarrow$ (7.3.3), by (6.3), and (7.3.3) $\Leftrightarrow$ (7.3.2), by (6.5) and (6.8). Finally, if $R$ is quasi-unmixed, then depth $w=$ altitude $R$, and $R / w$ satisfies the altitude formula for all $w \in$ mAss $R$. Also, $M \in \hat{A}^{*}(I)$ implies $M / w \in \hat{A}^{*}((I+w) / w)$ for some $w \in \mathrm{mAss} R$, by (6.3.1). Therefore $l((I+w) / w)=\operatorname{depth} w=$ altitude $R$, by [6, Thm. 3], and altitude $R \geq l(I) \geq l((I+w) / w)$, by [2] and (7.2), so $(7.3 .2) \Rightarrow(7.3 .1)$. 
(7.4) sharpens [6, Thm. 3], proves the converse of that result, and also extends the result from the Noetherian domain case.

(7.4) Corollary. Let $I \subseteq P$ be ideals in a Noetherian ring $R$ such that $P$ is prime. If $l\left(I_{P}\right)=$ height $P$, then $P \in \hat{A}^{*}(I)$. The converse holds if $R$ is locally quasi-unmixed.

Proof. If $l\left(I_{P}\right)=$ height $P$, then $P_{P} \in \hat{A}^{*}\left(I_{P}\right)$, by (7.3.1) $\Rightarrow$ (7.3.2), so $P \in \hat{A}^{*}(I)$. If $R$ is locally quasi-unmixed and $P \in \hat{A}^{*}(I)$, then $P_{P} \in \hat{A}^{*}\left(I_{P}\right)$ and $R_{P}$ is quasi-unmixed, so $l\left(I_{P}\right)=$ height $P$, by (7.3.2) $\Rightarrow(7.3 .1)$.

This paper will be closed by using (7.3) to give another proof of part of $[15$, Thm. 1].

(7.5) COROllary. ( $C f .[15, T h m .1]$.$) Let (R, M)$ be a local ring such that altitude $R \geq 1$. Then there exists a depth one $z \in \mathrm{mAss} R^{*}$ if and only if $M \in \hat{A}^{*}(I)$ for all ideals $I$ in $R$ such that height $I \geq 1$.

Proof. Assume first that there exists a depth one $z \in \mathrm{mAss} R^{*}$. Now for all ideals $I$ in all local rings $L$ it holds that altitude $L \geq l(I) \geq$ height $I$, by [2] and [9, Lemma 4, p. 151], so the conclusion follows immediately from (7.3.5) $\Rightarrow$ (7.3.2).

Conversely, if $M \in \hat{A}^{*}(b R)$ for some height one principal ideal $b R$, then (7.3.2) $\Rightarrow(7.3 .5)$ implies that there exists a depth one $z \in \mathrm{mAss} R^{*}$, since $\left(b R^{*}+z\right) / z$ is principal and $l(I) \leq v(I)=$ the minimal number of generators of $I$, by $[9$, Lemma 4 , p. 151].

\section{REFERENCES}

[1] M. Brodmann, Asymptotic stability of $\operatorname{Ass}\left(M / I^{n} M\right)$, Proc. Amer. Math. Soc., 74 (1979), 16-18.

[2] L. Burch, Codimension and analytic spread, Math. Proc. Cambridge Philos. Soc., 72 (1973), 369-373.

[3] $\quad$ A theorem on integral closures of ideals in semi-local rings, Math. Proc. Cambridge Philos. Soc., 95 (1979), 55-59.

[4] S. McAdam, Saturated chains in Noetherian rings, Indiana Univ. Math. J., 23 (1974), 719-728.

[5] S. McAdam and P. Eakin, The asymptotic Ass, J. Algebra, 61 (1979), 71-81.

[6] _ Asymptotic prime divisors and analytic spreads, Proc. Amer. Math. Soc., 80 (1980), 555-559.

[7] Asymptotic Prime Divisors, Lecture Notes in Math., Springer-Verlag, New York (to appear).

[8] M. Nagata, Local Rings, Interscience Tracts, No. 13, Interscience Publ., New York, 1961. 
[9] D. G. Northcott and D. Rees, Reductions of ideals in local rings, Math. Proc. Cambridge Philos. Soc., 50 (1954), 145-158.

[10] L. J. Ratliff, Jr., A characterization of analytically unramified semi-local rings, and applications, Pacific J. Math., 27 (1968), 127-143.

[11] On quasi-unmixed local domains, and altitude formula, and the chain condition for prime ideals (I), Amer. J. Math., 91 (1969), 508-528.

[12] - On prime divisors of the integral closure of a principal ideal, J. Reine Angew. Math., 255 (1972), 210-220.

[13] Locally quasi-unmixed Noetherian rings and ideals of the principal class, Pacific J. Math., 52 (1974), 185-205.

[14] _ On prime divisors of $I^{n}$, $n$ large, Michigan Math. J., 23 (1976), 337-352.

[15] _ Integrally closed ideals and asymptotic prime divisors, Pacific J. Math., 91 (1980), 445-456.

[16] Independent elements, integrally closed ideals, and quasi-unmixedness, J. Algebra, (to appear).

[17] __ Asymptotic sequences, J. Algebra, (to appear).

[18] _ A brief survey and history of asymptotic prime divisors, Rocky Mountain J. Math., (to appear).

[19] D. Rees, The grade of an ideal or module, Math. Proc. Cambridge Philos. Soc., 53 (1957), 28-42.

[20] __ A note on form rings and ideals, Mathematika, 4 (1957), 51-60.

[21] _ Rings associated with ideals and analytic spread, Math. Proc. Cambridge Philos. Soc., 89 (1981), 423-432.

[22] K. Whittington, Asymptotic prime divisors and the altitude formula, 20-page preprint.

[23] O. Zariski and P. Samuel, Commutative Algebra, Vol. I, Van Nostrand, New York, 1958.

[24] Commutative Algebra, Vol. II, Van Nostrand, New York, 1960.

Received June 11, 1982 and in revised form August 10, 1982. Research on this paper was supported in part by the National Science Foundation, Grant MCS80-01597-A01. 



\title{
PACIFIC JOURNAL OF MATHEMATICS EDITORS
}

\author{
Donald BABBITT (Managing Editor) \\ University of California \\ Los Angeles, CA 90024 \\ Hugo RossI \\ University of Utah \\ Salt Lake City, UT 84112 \\ C. C. MOore and Arthur Ogus \\ University of California \\ Berkeley, CA 94720
}

J. DUGUNDJI
Department of Mathematics

University of Southern California

Los Angeles, CA 90089-1113

R. FinN and H. SAMELSON

Stanford University

Stanford, CA 94305

\section{ASSOCIATE EDITORS}
R. ARENS
E. F. BECKENBACH
B. H. NeumanN
F. WOLF
K. YosHIDA (1906-1982)

\section{SUPPORTING INSTITUTIONS}

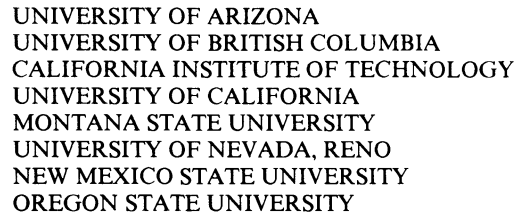

UNIVERSITY OF ARIZONA

UNIVERSITY OF BRITISH COLUMBIA

CALIFORNIA INSTITUTE OF TECHNOLOGY

UNIVERSITY OF CALIFORNIA

MONTANA STATE UNIVERSITY

UNIVERSITY OF NEVADA, RENO

NEW MEXICO STATE UNIVERSITY

OREGON STATE UNIVERSITY

\author{
UNIVERSITY OF OREGON \\ UNIVERSITY OF SOUTHERN CALIFORNIA \\ STANFORD UNIVERSITY \\ UNIVERSITY OF HAWAII \\ UNIVERSITY OF TOKYO \\ UNIVERSITY OF UTAH \\ WASHINGTON STATE UNIVERSITY \\ UNIVERSITY OF WASHINGTON
}

The Supporting Institutions listed above contribute to the cost of publication of this Journal, but they are not owners or publishers and have no responsibility for its content or policies.

Mathematical papers intended for publication in the Pacific Journal of Mathematics should be in typed form or offset-reproduced (not dittoed), double spaced with large margins. Please do not use built up fractions in the text of the manuscript. However, you may use them in the displayed equations. Underline Greek letters in red, German in green, and script in blue. The first paragraph must be capable of being used separately as a synopsis of the entire paper. In particular it should contain no bibliographic references. Please propose a heading for the odd numbered pages of less than 35 characters. Manuscripts, in triplicate, may be sent to any one of the editors. Please classify according to the scheme of Math. Reviews, Index to Vol. 39. Supply name and address of author to whom proofs should be sent. All other communications should be addressed to the managing editor, or Elaine Barth, University of California, Los Angeles, California 90024.

There are page-charges associated with articles appearing in the Pacific Journal of Mathematics. These charges are expected to be paid by the author's University, Government Agency or Company. If the author or authors do not have access to such Institutional support these charges are waived. Single authors will receive 50 free reprints; joint authors will receive a total of 100 free reprints. Additional copies may be obtained at cost in multiples of 50 .

The Pacific Journal of Mathematics is issued monthly as of January 1966. Regular subscription rate: $\$ 132.00$ a year (6 Vol., 12 issues). Special rate: $\$ 66.00$ a year to individual members of supporting institutions.

Subscriptions, orders for numbers issued in the last three calendar years, and changes of address should be sent to Pacific Journal of Mathematics, P.O. Box 969, Carmel Valley, CA 93924, U.S.A. Old back numbers obtainable from Kraus Periodicals Co., Route 100, Millwood, NY 10546.

The Pacific Journal of Mathematics ISSN 0030-8730 is published monthly by the Pacific Journal of Mathematics at P.O. Box 969, Carmel Valley, CA 93924. Application to mail at Second-class postage rates is pending at Carmel Valley, California, and additional mailing offices. Postmaster: Send address changes to Pacific Journal of Mathematics, P. O. Box 969, Carmel Valley, CA 93924.

PUBLISHED BY PACIFIC JOURNAL OF MATHEMATICS, A NON-PROFIT CORPORATION 


\section{Pacific Journal of Mathematics}

\section{Vol. 111, No. $2 \quad$ December, 1984}

Berndt Brenken, Representations and automorphisms of the irrational

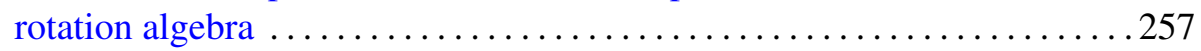

Harold George Diamond, A number theoretic series of I. Kasara . . . . . . 283

Rolf Farnsteiner, On the structure of simple-semiabelian Lie algebras . . . . 287

Guillermo Grabinsky, Poisson process over $\sigma$-finite Markov chains . ......301

Derbiau Frank Hsu and A. Donald Keedwell, Generalized complete

mappings, neofields, sequenceable groups and block designs. I . . . . . 317

William H. Julian and Fred Richman, A uniformly continuous function on

$[0,1]$ that is everywhere different from its infimum $\ldots \ldots \ldots \ldots . \ldots 33$

D. H. Lehmer and Emma Lehmer, The sextic period polynomial .........341

E. Maluta, Uniformly normal structure and related coefficients ...........357

Coy Lewis May, The species of bordered Klein surfaces with maximal

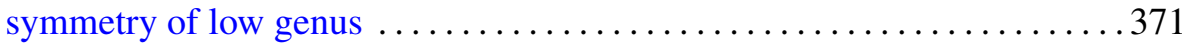

Louis Jackson Ratliff, Jr., On asymptotic prime divisors . . . . . . . . . . . 395

Norbert Riedel, Disintegration of KMS-states and reduction of standard von

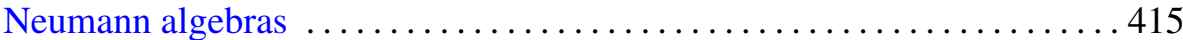

Richard Gordon Swan, $n$-generator ideals in Prüfer domains ...........433

Vilmos Totik, An interpolation theorem and its applications to positive

operators .................................. 447

Richard Vrem, Hypergroup joins and their dual objects 